\title{
PRIMER REGISTRO DE PHARYPIA PULCHELLA (DRURY, 1782) (HEMIPTERA: PENTATOMIDAE) PARA LA REPÚBLICA DOMINICANA Y LA ESPAÑOLA
}

\author{
Gabriel de los Santos ${ }^{1}$, Ruth H. Bastardo ${ }^{2}$ y Daniel E. Perez-Gelabert ${ }^{3}$ \\ ${ }^{1}$ Museo Nacional de Historia Natural "Prof. Eugenio de Jesús Marcano". Calle César Nicolás Penson, \\ Plaza de la Cultura, Santo Domingo, 10204, República Dominicana.g.delossantos@mnhn.gov.do \\ ${ }^{2}$ Instituto de Investigaciones Botánicas y Zoológicas, Profesor Rafael M. Moscoso. \\ Universidad Autónoma de Santo Domingo, Santo Domingo, 10105, \\ República Dominicana.r bastardo@hotmail.com \\ ${ }^{3}$ Integrated Taxonomic Information System (ITIS) and Department of Entomology, \\ National Museum of Natural History, Smithsonian Institution, P. O. Box 37012, \\ Washington, D. C. 20013-7012, USA.perezd@si.edu
}

\section{RESUMEN}

Se registra por primera vez para la República Dominicana y La Española el pentatómido Pharypia pulchella (Drury, 1782). Con este registro se eleva a 78 para la República Dominicana y 80 para la isla el total de especies de Pentatomidae reportadas.

Palabras clave: Pentatomidae, Pharypia, República Dominicana, La Española, nuevo registro.

Title: First record of Pharypia pulchella (Drury, 1782) (Hemiptera: Pentatomidae) from the Dominican Republic and Hispaniola.

\section{ABSTRACT}

The stink bug species Pharypia pulchella (Drury, 1782) is recorded for the first time from Dominican Republic and Hispaniola. With this record the total number of Pentatomidae species recorded from the Dominican Republic is increased to 78, while the number for the whole island is raised to 80 .

Keywords: Pentatomidae, Pharypia, Dominican Republic, Hispaniola, new record.

La familia Pentatomidae (Hemiptera) posee reconocida importancia económica debido a que algunas de sus especies son consideradas plagas agrícolas. No obstante, al ser depredadores, los miembros de la subfamilia Asopinae presentan potencial como control biológico (Thomas, 1992; Costello et al., 2002).

A nivel mundial, la familia Pentatomidae incluye 4,722 especies válidas comprendidas en 896 géneros (Rider, 2011). En la República Dominicana la fauna de "hiedevivos", como se les conoce localmente, ha recibido atención en la última década. Perez-Gelabert y Thomas (2005), inventariaron 77 especies para La Española. Este trabajo incluye la descripción de siete nuevas especies para la ciencia a partir de poblaciones de República Dominicana, así como la discusión de algunos caracteres de identificación, plantas hospederas, distribución y una lista de especies. La publicación citada es la más amplia que se ha realizado sobre esta familia en República Dominicana, la misma sirvió de base para la revisión de dos colecciones del grupo en este país (de los Santos, 2012) y dos publicaciones posteriores (de los Santos y Bastardo, 2012; 2013).

La presencia de Pharypia pulchella (Drury) en la República Dominicana fue considerada como "dudosa" por de los Santos (2012: 28) y de los Santos y Bastardo (2013), ya que el único 
ejemplar que se encontró en la colección del Instituto de Investigaciones Botánicas y Zoologícas, Profesor Rafael M. Moscoso (IIBZ) de la Universidad Autónoma de Santo Domingo, República Dominicana, carecía de la información de colecta. Sin embargo, debido a que existen registros de esta especie en Puerto Rico, los referidos autores dejaron abierta la posibilidad de su presencia en la República Dominicana. Recientemente, llegó un ejemplar al IIBZ (Fig. 1A-B), el cual pertenece a $P$. pulchella (Drury), representando este el primer registro de esta especie para la República Dominicana y La Española.

\section{TAXONOMÍA}

Familia Pentatomidae Leach, 1815

Subfamilia Pentatominae Leach, 1815

Tribu Pentatomini Leach, 1815

Género Pharypia Stål, 1861

Pharypia pulchella (Drury, 1782)
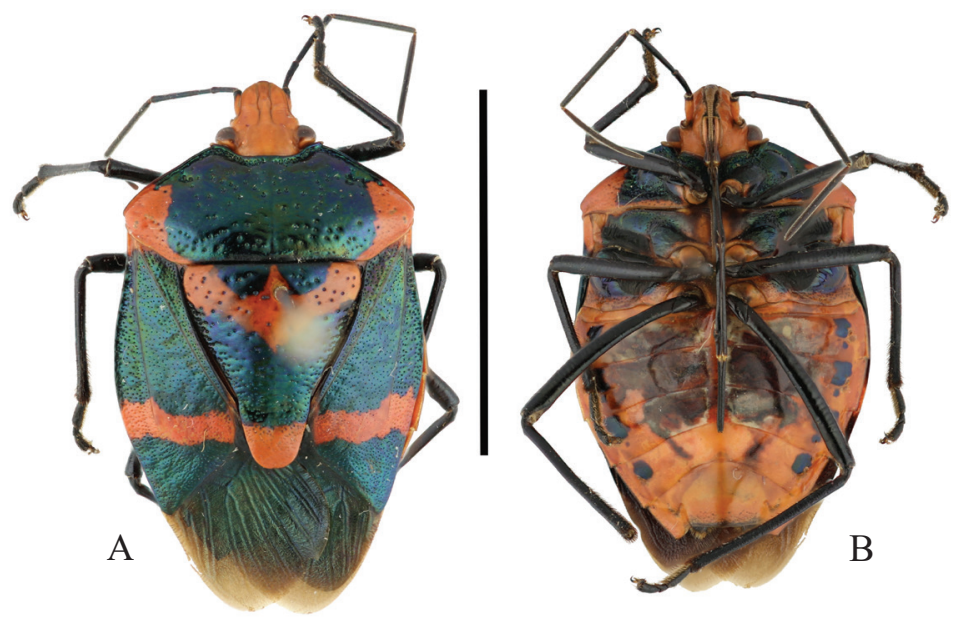

Figura 1, A-B. Pharypia pulchella (Drury, 1782). A, vista dorsal. B, vista ventral. Escala: $10 \mathrm{~mm}$.

Reconocimiento. Pentatómidos medianos a grandes (14-18 mm), con llamativa coloración aposemática, donde la cabeza es rojo-anaranjada, al igual que dos bandas delgadas transversales bien separadas sobre el cuerpo de color verde negruzco metálico iridiscente. Una banda anterior en forma de "U" parte de los ángulos humerales y se extiende hasta la parte media del escutelo (Fig. 1A); la otra banda atraviesa el cuerpo horizontalmente al nivel del ápice escutelar. Ventralmente el cuerpo es principalmente anaranjado con variables manchas negras en su porción anterior (Fig. 1B).

Comentarios. El género Pharypia Stål, 1861, incluye seis especies (Rider, 2005) que se distribuyen en la Región Neotropical, siendo Pharypia pulchella la única que tiene una amplia distribución en el neotrópico. Esta especie fue colectada en Costa Rica por Nielsen et al. (2004), alimentándose de las plantas: Genipa americana y Randia subcordata (Rubiaceae), Picramnia sp. (Simaroubaceae), Acalypha diversifolia (Euphorbiaceae), Mouriri myrtilloides (Melastomataceae), Tetracera volubilis (Dilleniaceae) y Serjania sp. (Sapindaceae). Bailowitz y Palting (2010) la reportan asociada a varias especies de Randia spp. (Rubiaceae) en Sonora, México. 
La colección del National Museum of Natural History, Smithsonian Institution, Washington, D. C., contiene alrededor de 50 especímenes de P. pulchella provenientes de México, Guatemala, Panamá, Colombia y Puerto Rico. Dado que alrededor de la mitad de estos son de Puerto Rico, parecería que allí esta especie no es rara como en República Dominicana.

Material revisado. 1 (ð). República Dominicana, Santo Domingo Norte, Guanuma. 22-X-2014. Col., Ana María Rodríguez. [IIBZ].

Distribución. México, Guatemala, República Dominicana, Puerto Rico, Honduras, Costa Rica, Panamá, Colombia, Venezuela y Brasil.

\section{LITERATURA CITADA}

Bailowitz, R. A. y J. Palting. 2010. Biodiversidad de los insectos con especial énfasis en Lepidoptera y Odonata. En: F. E. Molina-Freaner y T. R. Van Devender, eds. Diversidad Biológica de Sonora. UNAM, México, pp. 315-337.

Costello, S. L., P. D. Pratt, M. B. Rayachhetry y T. D. Center. 2002. Morphology and life history characteristics of Podisus mucronatus (Heteroptera: Pentatomidae). Florida Entomologist, 85: 344-350.

De los Santos, G. 2012. Los Pentatómidos (Insecta: Hemiptera: Heteroptera: Pentatomidae) en las colecciones de referencia de la República Dominicana. Tesis de Licenciatura en Biología, Universidad Autónoma de Santo Domingo, República Dominicana. V + 105 pp.

De los Santos, G. y R. Bastardo. 2012. Nuevos registros de hiedevivos (Insecta: Hemiptera: Pentatomidae) para la República Dominicana y La Hispaniola, incluyendo el nuevo hallazgo de Piezosternum subulatum (Thunberg, 1783). Novitates Caribaea, 5: 120-123.

De los Santos, G. y R. Bastardo. 2013. La familia Pentatomidae (Hemiptera: Heteroptera) en las colecciones de referencia de la República Dominicana. Novitates Caribaea, 6: 1-15.

Nielsen, V., P. Hurtado, D. H. Janzen, G. Tamayo y A. Sittenfeld. 2004. Recolecta de artrópodos para prospección de la biodiversidad en el Área de Conservación Guanacaste, Costa Rica. Revista de Biología Tropical, 52: 119-132.

Perez-Gelabert, D. E. y D. B. Thomas. 2005. Stink bugs (Pentatomidae: Heteroptera) of the island of Hispaniola, with seven new species from the Dominican Republic. Boletín de la Sociedad Entomológica Aragonesa, 37: 319-352.

Rider, D. 2005. Pharypia Stål, 1861, Pentatomoidea Home Page. https://www.ndsu.edu/ pubweb/ rider/Pentatomoidea/Genus_Pentatomini/Pharypia.htm (19 de mayo, 2016).

Rider, D. 2011. Number of Genera \& Species, Pentatomoidea Home Page. http://www. ndsu.nodak.edu/ndsu/rider/Pentatomoidea/Classification/Genus_Species_Numbers.htm (19 de mayo, 2016).

Thomas, D. B. 1992. Taxonomic synopsis of the Asopine Pentatomidae (Heteroptera) of the Western Hemisphere. Thomas Say Monographs No. 16, Entomological Society of America, Lanham, Maryland, 156 pp. 